Z. klin. Chem. u. klin. Biochem.

8. Jg., S. $483-487$, September 1970

\title{
Fettsäuremuster der Rattenleber bei Verfütterung gerad- oder ungerad- zahliger mittelkettiger Triglyceride und verschiedener Linolsäuremengen
}

\author{
Von P. N. SENG ${ }^{1}$ ) und J. BERNǍšEK \\ Klinisch-Chemische Abteilung (Prof. Dr. K. D. Voigt) der II. Medizinischen Universitätsklinike \\ Hamburg-Eppendorf und Pbysiologisch-Chemisches Institut (Prof. Dr. H. Hilz) der Universität Hamburg
}

(Eingegangen am 24. April 1970)

Es wurden gaschromatographische Analysen der Lebergesamtfettsäuren von 6 Gruppen zu 9 bzw. 10 männlichen Ratten durchgeführt. Alle Tiere erhielten von der 4. Lebenswoche an für 8 Wochen eine fettfreie Grunddiät mit Zusatz von 10 Gewichtsprozent eines mittelkettigen Triglycerids. Drei der Gruppen erbielten $10 \%$ Tripelargonin von der 5 . bis zur 12. Lebenswoche, die übrigen drei Gruppen bekamen von der 5. bis zur 8. Lebenswoche ein gemischtes mittelkettiges Triglycerid, dessen Fettsäuren sich etwa je zur Hälfte aus Capryl- $\left(C_{8: 0}\right)$ und Caprinsäure $\left(C_{10: 0}\right)$ zusammensetzten. Ein reines Tricaprylin erhielten diese letzten 3 Gruppen von der 9 . bis zur 12 . Lebenswoche. Darüberhinaus erhielten je eine Gruppe mit dem ungeradzahligen und eine Gruppe mit dem geradzahligen mittelkettigen Triglycerid $1 \mathrm{Gew} \%$ Linolsäure, je eine weitere $0,2 \%$ Linolsäure, die jeweils 3. Gruppe erhielt keine Linolsäurc. Unterschiede in der Zusammensetzung der Leberfettsäuren der mit gerad- oder ungeradzahligem mittelkettigem Triglyceriden ernährten Ratten wurden

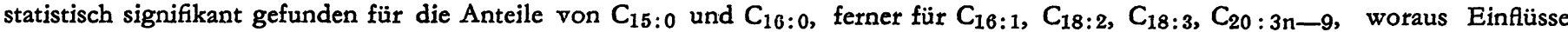
der verschiedenen mittelkettigen Triglycerid-Präparate auf den Stoffwechsel u. a. der essentiellen Fettsäuren abzulesen sind.

\section{The fatty acids of rat liver after diets of even or odd numbered medium chain triglycerides and varying amounts of linoleic acid}

The total liver fatty acids of six groups of 9 or 10 male rats were analysed by gas chromatography. Starting at the fourth week of life, each animal received a fat-free basic diet with the addition of $10 \%$ by weight of a medium chain triglyceride preparation for the following 8 weeks. Three of the groups received $10 \%$ tripelargonin from age 5 until 12 weeks; of these three groups, one received an additional $1 \%$ linoleic acid, the second $0.2 \%$ linoleic acid and the third received no linoleic acid. The remaining three groups received a mixed medium chain triglyceride, containing about one half each of caprylic $\left(C_{8: 0}\right)$ and capric acid $\left(C_{10: 0}\right)$, from age 5 until 8 weeks. These latter three groups received pure tricaprylin from age 9 until 12 weeks and they also differed in the addition of linoleic acid, i. e., $1 \%$, $0.2 \%$ or none, respectively.

The composition of the liver fatty acids from rats receiving even or odd numbered medium chain triglyceride showed statistically significant differences for $C_{15: 0}$ and $C_{16: 0}$ and also for $C_{16: 1}, C_{18: 2}, C_{18: 3}, C_{20}: 3 n-9$; this indicates that the different medium chain triglyceride preparations affect the metabolism of the fatty acids, including the essential fatty acids.

$\mathrm{Da}$ in den letzten Jahren geradzahlige mittelkettige Triglyceride ${ }^{2}$ ) in steigendem Maße diätetische Verwendung finden $(1-3)$ und auch die Verwendung ungeradzahliger Triglyceride aus guten Gründen in Erwägung gezogen werden kann (geringere Ketogenesel), erschien es uns interessant, die Auswirkungen ihrer Verabreichung auf die Organfettsäuren, etwa der Leber, vergleichend $\mathrm{zu}$ studieren.

\section{Material und Methoden}

Für Herstellung und Analyse des Tricaprylin und Tripelargonin danken wir den Herren Dipl. Chem. H. E. Rost und Dr. A. K. SEN GuPTA (Unilever Forschungslaboratorium Hamburg). Das geradzahlige gemischte mittelkettige Triglycerid stellte mit Analyse dankenswerterweise die Wiss. Abteilung der MargarineUnion Hamburg zur Verfügung (Tab. 1).

Es wurden männliche Sprague-Dawley-Ratten eines eigenen Inzuchtstammes verwendet. Die Muttertiere erhielten bis zum Tage des Absetzens Altromin $\mathbf{R}^{3}$ ) und Wasser ad libitum. Nach dem Absetzen am 28. Lebenstag erbielten die Versuchstiere die je-

1) Jetzige Adresse: Physiologisch-Chemisches Institut der Universität Köln, 5 Köln 41, Joseph-Stelzmann-Str. 52.

2) Sog. mittelkettige Triglyceride enthalten unverzweigte gesättigte Fettsäuren mit 6-12 C-Atomen in der Kette. Englisch: Medium Chain Triglycerides; abgekürzt MCT.

3) Altromin R: Hersteller Altromin GmbH. 491 Lage (Lippe), Postf. 285. Fett- und Fettsäurezusammensetzung des Altromin siche 1. c. (5). weilige Versuchskost ad libitum. Es wurden 6 verschiedene Diäten an 6 Gruppen zu je 10 bzw. 9 Tieren verfüttert. Alle erhielten die gleiche fettfreie Grunddiät in der Zusammensetzung der Tabelle 2 mit $10 \mathrm{Gew} \%$ mittelkettige Trigylceride. Je 3 Gruppen (I, III, V) erhielten geradzahliges oder ungeradzahliges mittelkettiges Triglycerid (Tab. 1) von der 5. bis zur 12. Lebenswoche. Während der letzten 3 Wochen wurde das gemischte geradzahlige mittelkettige Triglycerid ersetzt durch reines Tricaprylin, während als ungeradzahliges mittelkettiges Triglycerid immer reines Tripelargonin zur Verfügung stand. Je eine

Tab. 1

Zusammensetzung der verfütterten mittelkettigen TriglyceridPräparate (MCT)

Glyceride in Flächenprozent nach Gaschromatographie Unverseifbares in Gewichtsprozent

Geradzahliges gemischtes mittelkettiges Trigylcerid (5.-8. Woche Gruppen I, III, V)

Triglyceride

$8 / 8 / 8$

$8 / 8 / 10$

8/10/10

Diglyceride

$8 / 810 \mathrm{H}$

$8 / 10 / \mathrm{OH}$

$10 / 10 / 0 \mathrm{H}$

Unverseifbares

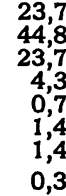

Tricaprylin (9.-12. Woche Gruppen I, III, V)

$\begin{array}{lcr}\text { Triglycerid } & 8 / 8 / 8 & 97,8 \\ \text { Diglycerid } & 8 / 8 / 0 \mathrm{H} & 1,9 \\ \text { Sonstige Glyceride } & & 0,3 \\ \text { Unverseifbares } & & 0,06\end{array}$

.Tripelargonin (5.-12. Woche Gruppen II, IV, VI)
Triglycerid
Diglycerid
Sonstige Glyceride
9/ $9 / 9$
93,2
6,3
0,5
Unverseifbares 
der Gruppen mit gerad- oder ungeradzahligem mittelkettigem Triglycerid bekam $1 \mathrm{Gew} \%$ Linolsäure (Gruppen I und II), je eine weiterc $0,2 \%$ Linolsäure (Gruppen III und IV) und die jeweils letzte Gruppe (V und VI) keine Linolsäure.

Entsprechend dem nach unseren Analysen etwa 20proz. Anteil der Nicht-Linolsäure-Bestandteile im verfütterten Linolsäurepräparat ${ }^{4}$ )

Tab. 2

Futteranalyse

A. Zusammensetzung des Diätfutters (Gewichtsprozent) Vitamine (einschl. Rohrzucker) Mineralien

Cellulose

Mittelkettige Triglyceride (gerad- oder ungeradzahlig) Casein (Äthanol gewaschen)

Stärke

56,$25 ; 57,25 ;$ oder

Linolsäure-Präparat

1,$25 ; 0,25$; oder

B. Zusammensetzung der fettlöslichen Vitamine pro $1 \mathrm{~kg}$ Futter

Vitamin $D_{2} 2000 \mathrm{I}$. E. Liponsäure $30 \mathrm{mg}$

Tocopherol $300 \mathrm{mg}$ Ubichinon $15 \mathrm{mg}$

Vitamin A $50 \mathrm{mg} \quad$ Vitamin $\mathrm{K}_{3} \quad 10 \mathrm{mg}$

C. Zusammensetzung der wasserlöslichen Vitamine pro $1 \mathrm{~kg}$ Futter

$\begin{array}{llll}\text { Cholinchlorid } & 1,5 \mathrm{~g} & \text { Niacin } & 0,06 \mathrm{~g} \\ \text { DL-Methionin } & 1,2 \mathrm{~g} & \text { Ca-Pantothenat } & 0,08 \mathrm{~g}\end{array}$

DL-Methionin $1,2 \mathrm{~g}$

$\begin{array}{llll}\text { meso-Inosit } & 1,0 \mathrm{~g} & \text { Thiaminchlorid } & 0,06 \mathrm{~g} \\ \text { Folsäure } & 0,03 \mathrm{~g} & \text { Riboflavin } & 0,03 \mathrm{~g}\end{array}$

Pyridoxin

benzoesäure $0,3 \mathrm{~g}$

Orotsäure $0,1 \mathrm{~g}$

$\begin{array}{ll}\text { Rutin } & 0,2 \xi ్ \\ & 0,006\end{array}$

Biotin

Vitamin $B_{1}$

Rohrzucker ad

$0,02 \mathrm{~g}$

$0,002 \mathrm{~g}$

$20,0 \mathrm{~g}$

D. Zusammensetzung von Mineralien und Spurenelementen pro $1 \mathrm{~kg}$ Futter

\begin{tabular}{|c|c|c|c|}
\hline $\begin{array}{l}\mathrm{Ca} \mathrm{CO} \\
\text { Tri-Ca-Phosphat } \\
\mathrm{K}_{2} \mathrm{HPO}_{4} \\
\mathrm{NaCl} \\
\mathrm{Na}_{2} \mathrm{HPO}_{4} \\
\mathrm{MgSO}_{4} \cdot 7 \mathrm{H}_{2} \mathrm{O} \\
\mathrm{FeII}_{4} \mathrm{Gluconat}_{2} \cdot 2 \mathrm{H}_{2} \mathrm{O} \\
\mathrm{MnSO}_{4} \cdot 4 \mathrm{H}_{2} \mathrm{O} \\
\mathrm{CuSO} \cdot 5 \mathrm{H}_{2} \mathrm{O}\end{array}$ & $\begin{array}{r}10,0 \mathrm{~g} \\
5,0 \mathrm{~g} \\
9,0 \mathrm{~g} \\
8,0 \mathrm{~g} \\
7,0 \mathrm{~g} \\
2,0 \mathrm{~g} \\
3,0 \mathrm{~g} \\
0,5 \mathrm{~g} \\
0,03 \mathrm{~g}\end{array}$ & $\begin{array}{l}\mathrm{ZnCO}_{3} \\
\mathrm{NaF} \\
\mathrm{KJ} \\
\mathrm{KBr} \\
\mathrm{As}_{2} \mathrm{O}_{3} \\
\mathrm{H}_{3} \mathrm{BO}_{3} \\
\mathrm{CoCl}_{2} \cdot 6 \mathrm{H}_{2} \mathrm{O} \\
\mathrm{AlNH}_{4}\left(\mathrm{SO}_{4}\right) \cdot 12 \mathrm{H}_{2} \mathrm{O} \\
\mathrm{Na}_{2} \mathrm{MOO}_{4} \cdot 2 \mathrm{H}_{2} \mathrm{O}\end{array}$ & $\begin{array}{r}30,0 \mathrm{mg} \\
3,0 \mathrm{mg} \\
3,0 \mathrm{mg} \\
0,8 \mathrm{mg} \\
0,3 \mathrm{mg} \\
0,3 \mathrm{mg} \\
0,1 \mathrm{mg} \\
1,0 \mathrm{mg} \\
0,2 \mathrm{mg}\end{array}$ \\
\hline
\end{tabular}

Tab. 3

Zusammensetzung des verfütterten Linolsäurepräparates Molprozent der freien Fettsäuren nach Gaschromatographie der Methylester (siehe Methoden)

Unverseifbares und oxydierte Fettsäuren in Gewichtsprozent*)

\begin{tabular}{rrrr}
\hline$C_{12: 0}$ & 0,1 & $C_{18: 0}$ & 0,4 \\
$14: 0$ & 0,1 & $18: 1$ & 13,1 \\
$16: 0$ & 1,5 & $18: 2$ & 83,8 \\
$16: 1$ & 0,1 & $18: 3$ & 0,9 \\
Unverseifbares & 0,15 & & \\
Oxydierte Fettsäuren & 1,07 & & \\
\hline
\end{tabular}

*) analysiert vom Unilever Forschungslabor Hamburg

4) Linolsäure techn., Best.-Nr. 2-62240, Fa. Carl Roth oHG., 75 Karlsruhe.
(Tab. 3) wurde dieses nach Tabelle 2 dem Futter zugemischt im Austausch gegen Stärke.

Die Aufzucht erfolgte bei $22^{\circ}$ Raumtemperatur in Kunststoffkäfigen mit Drahtdeckeln. Die Tiere wurden wöchentlich gewogen.

Am Ende der 12. Lebenswoche wurden die Tiere durch Dekapitieren und Ausbluten getötet (ohne daß sie vorher einer Fastenperiode unterworfen wurden). Die Lebern wurden sofort entnommen, ein gewogener Teil von etwa $1-2 \mathrm{~g}$ unmittelbar in $4^{\circ}$ kaltem Chloroform-Methanol 2:1 ( $\left./ / v\right)$ homogenisiert und extrahiert (4). Die mit $0,1 \mathrm{M} \mathrm{KCl}$ géwaschenen Extrakte wurden am Rückflußkühler $1 \mathrm{Std}$. mit methanolischer $2 \mathrm{~N} \mathrm{KOH}(5,6)$ verseift, das Unverseifbare mit Petroläther ausgeschüttelt, nach Ansäuern der Unterphase mit $\mathrm{HCl}$ die Fettsäuren extrahiert und mit wasserfreier methanolischer 3proz. $\mathrm{H}_{2} \mathrm{SO}_{4}$ (1 Std. am Rückfluß) zu Methylestern umgesetzt. Die wiederum durch Petrolätherextraktion gewonnenen Fettsäuremethylester wurden gaschromatographisch analysiert, auf einem Gaschromatographen der Fa. Bodenseewerk Perkin-Elmer mit $2 \mathrm{~m}$ Edelstahlsäule, inn. Durchm. 2,7 mm, gepackt mit 2,5\% EGS auf Chromosorb G (80-100 mesh DMCSAW), isothermal bei $160^{\circ}$ Ofen-, $300^{\circ}$ Einspritz-Temperatur, unter einem Trägergasstrom von $25 \mathrm{~m} / / \mathrm{Min}$. $\mathrm{N}_{2}$ bei $2,5 \mathrm{kp} / \mathrm{cm}^{2}$ Druckabfall, mit Flammenionisationsdetektor. Ausgewertet wurden die auf einem Schreiber (Kompensograph $\mathrm{L} 288 \times 288$ der Fa. Siemens) registrierten Gaschromatogramme nach Einzeichnen der Basislinie und der Wendetangenten durch Multiplikation der Peakhöhe mit dem Peakbasisabschnitt zwischen den Schnittpunkten der Wendetangenten mit der Basislinie (7). Unter Berücksichtigung von empirisch nach 1. c. (5) ermittelten Eichfaktoren wurden die Anteile der einzelnen Komponenten an den Gesamtfettsäuren der Leber in Mol \% berechnet. Die Identifizierung erfolgte anhand der „Kohlenstoffzahl" (8) oder ECL (equivalent chain length) (9) durch Vergleich der Probenpeaks mit Standardsubstanzen (freundlicherweise zur Verfügung gestellt von Unilever Research Laboratories Vlaardingen, Holland). Standards für die Fettsäuremethylester $\mathrm{C}_{22: 4 \mathrm{n}-6}$ und $C_{22: 5 n-6}$ standen nicht zur Verfügung, so $\mathrm{da} \beta$ deren Identifizierung hypothetisch blieb, selbst unter Verwendung von Vergleichsliteratur $(10 ; 11)$. Die statistische Auswertung der Ergebnisse erfolgte im t-Test nach Student (Tab. aus (12)).

\section{Ergebnisse}

In Tabelle 4 finden sich die Mittelwerte $(x \pm s)$ für die Mol\%-Anteile der Einzelfettsäuren an den Gesamtfettsäuren der Leber bei den 6 Diätgruppen, in Tabelle 5 eine Zusammenstellung der P-Werte der Unterschiede nach statistischer Auswertung. Es traten Unterschiede

Tab. 4

Fettsäuren der Leber in Molprozent (Mittelwerte $\pm 1 \mathrm{~s}$ )

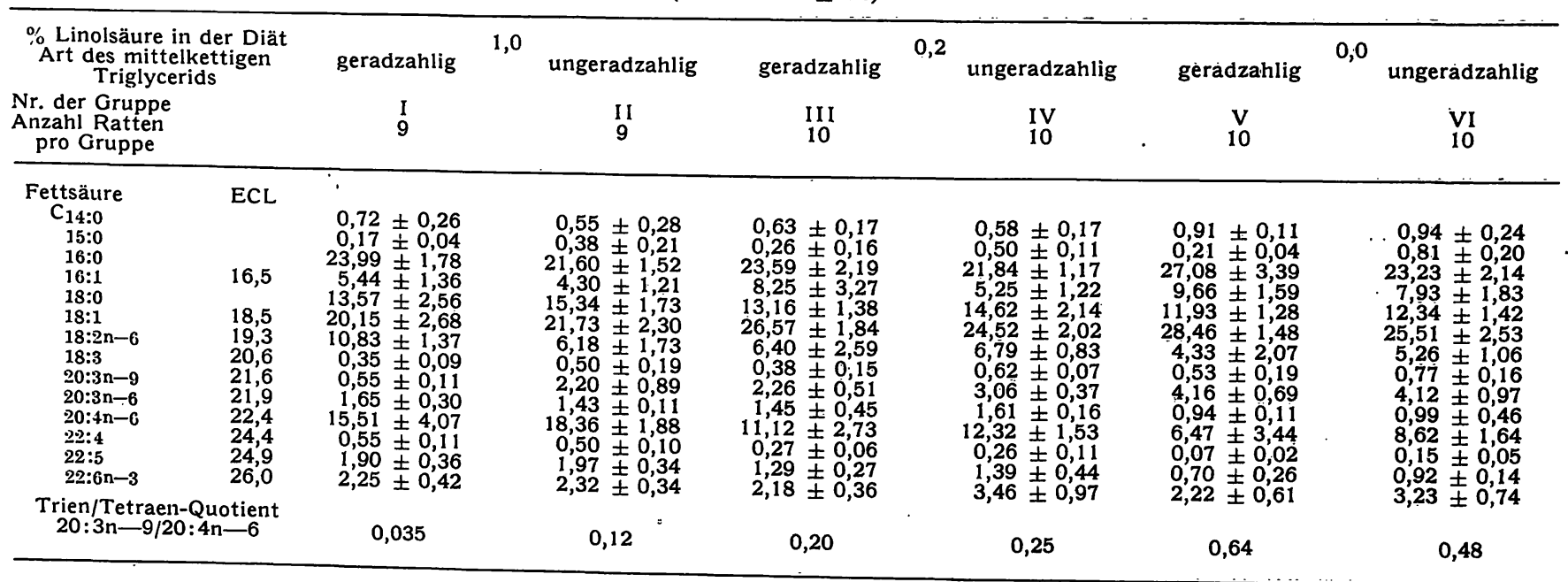


Störungen in der Vorphase der Gerinnung
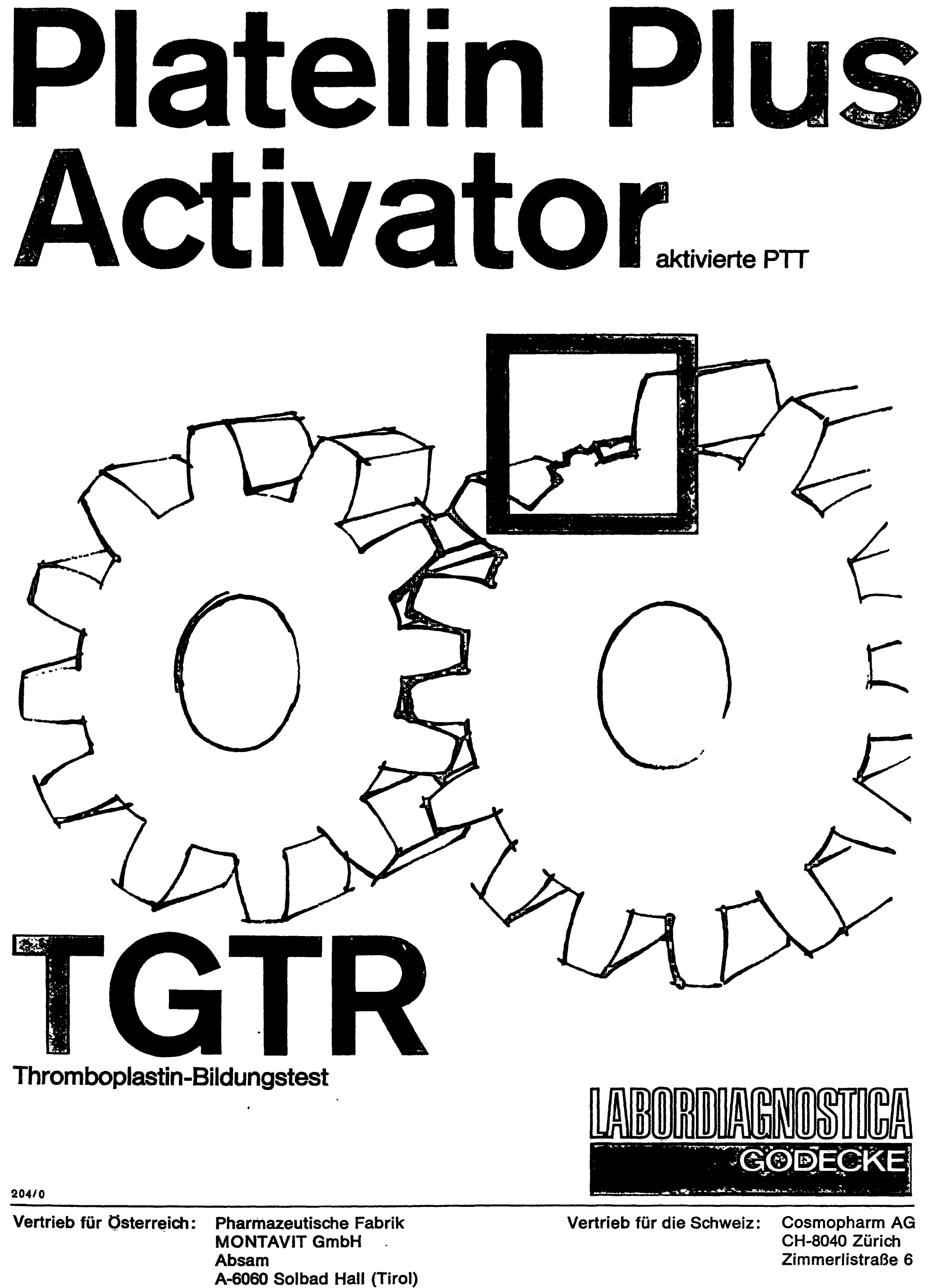


\section{Sie erfahren viel über das automatische SORVALL Zellwasch-System, auch über Coombs-Tests und andere serolog. Untersuchungen}
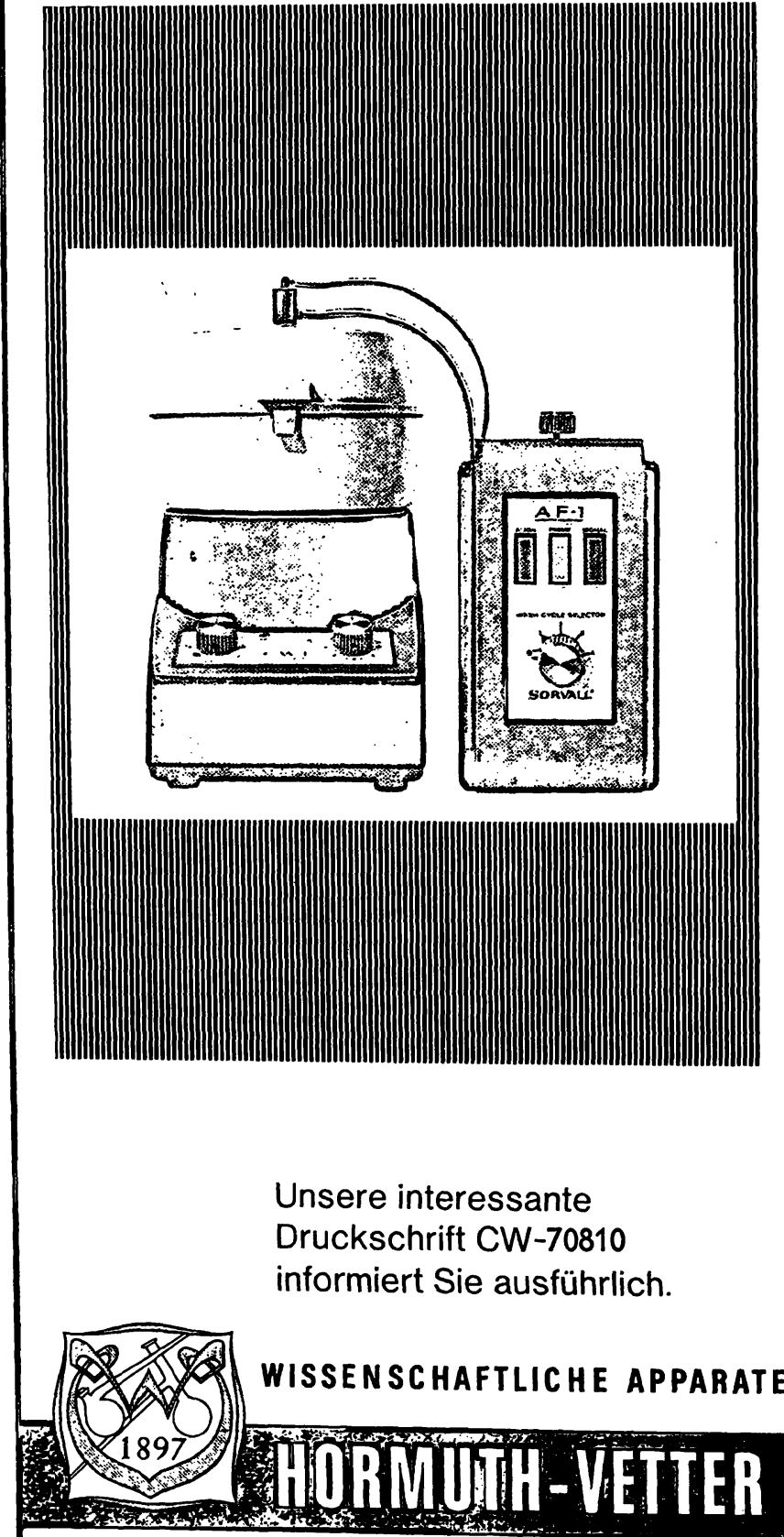

6908 Wiesloch/Bd., Postfach 1348, Tel. 06222/2147 6900 Heidelberg 1, Postfach 750, Tel. $06221 / 20045$
THURGAUISCHES KANTONSSPITAL MÜNSTERLINGEN

Im Zusammenhang mit unserem Spitalneubau soll die Stelle eines

\section{Leiters}

\section{des zentralen Laboratoriums}

geschaffen werden. - Wir möchten, daß unser zukünftiger Leiter schon während der Einrichtungsphase der Laboratorien mitberaten kann und sind daher sehr interessiert, schon heute mit einem geeigneten Anwärter eine entsprechende Vereinbarung zu treffen.

Interessenten mit medizinischer, chemischer oder pharmazeutischer Ausbildung bitten wir, mit unserer ärztlichen Direktion Kontakt aufzunehmen.

Thurgauisches Kantonsspital CH 8596 Münsterlingen (Schweiz)

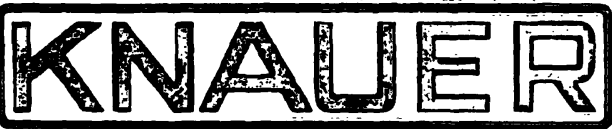

Komplettes System zur Molekulargewichtsbestimmung und Bestimmung der Osmolalität biologischer Lösungen
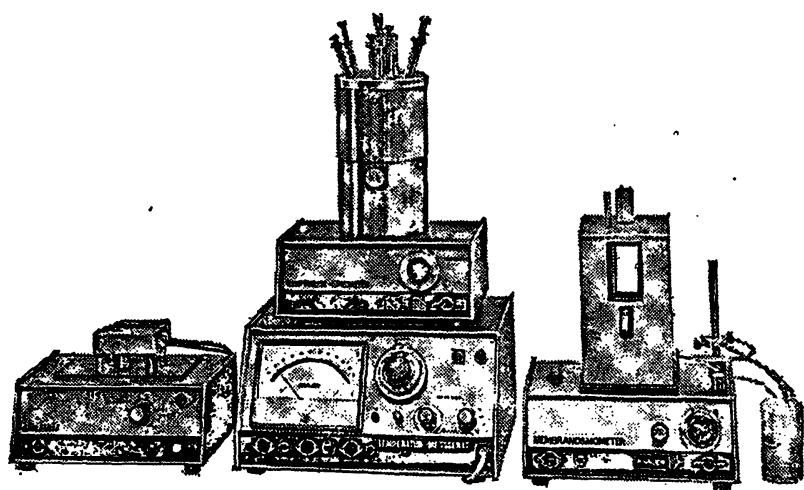

bestehend aus:

1 elektronischen Universal-Temperafur-Meßgerät Auflösungsvermögen $1 / 10000^{\circ} \mathrm{C}$

1 Dampfdruck-Osmometer-Zusatz für Molekulargewichte zwischen 50 und 25000 , neves Modell

DM 3080,DM 3100,DM 940,-

1 Elekolekirischen Kühlgerät zur Halbmikro-Kryoskopie

tronischen Membran-Osmometer-Zusatz für Molekulargewichte zwischen 10000 und 1000000 , MeBbereiche DM 4510, Alle Preise verstehen sich zuzüglich Mehrwertsfever.

KG Dr. Herbert KNAUER - 1 Berlin 37 (West) - Holstweg 18 Tel. (0311) 848705

Wir stellen aus: Chemie-70, 10.24. 9. 1970 Moskav, Areal Glahé International, Pavillon 20/21, Stand 15 
Tab. 5

P-Werte der Differenzen in den Fettsăuremustern (P-Werte $\geq 0,05$ wurden weggelassen)

\begin{tabular}{|c|c|c|c|c|c|c|c|c|c|}
\hline \multirow[b]{2}{*}{$\begin{array}{l}\text { Vergleich der } \\
\text { Gruppen Nr. } \\
\text { \% Linolsäure } \\
\text { der Diät }\end{array}$} & \multicolumn{3}{|c|}{$\begin{array}{l}\text { mittelkettige Triglyceride verschieden } \\
\text { Linolsäurezufuhr gleich }\end{array}$} & \multicolumn{3}{|c|}{$\begin{array}{c}\text { mittelkettige Triglyceride geradzahlig } \\
\text { Linolsäurezufuhr verschieden }\end{array}$} & \multicolumn{3}{|c|}{$\begin{array}{c}\text { mittelkettige Triglyceride ungeradzahlig } \\
\text { Linolsäurezufuhr verschieden }\end{array}$} \\
\hline & $\begin{array}{c}1-11 \\
1,0-1,0\end{array}$ & $\begin{array}{l}I 1 I-I V \\
0,2-0,2\end{array}$ & $\begin{array}{r}V-V I \\
0,0-0,0\end{array}$ & $\begin{array}{r}1-111 \\
1,0-0,2\end{array}$ & $\begin{array}{c}I-V \\
1,0-0,0\end{array}$ & $\begin{array}{l}111-V \\
0,2-0,0\end{array}$ & $\begin{array}{l}I I-I V \\
1,0-0,2\end{array}$ & $\begin{array}{r}I I-V I \\
\quad 1,0-0,0\end{array}$ & $\begin{array}{l}I V-V I \\
0,2-0,0\end{array}$ \\
\hline $\begin{array}{c}\text { Fettsäure } \\
C_{14: 0} \\
15: 0 \\
16: 0 \\
16: 1 \\
18: 0 \\
18: 1 \\
18: 2 \\
18: 3 \\
20: 3 n-9 \\
20: 3 n-8 \\
20: 4 \\
22: 4 \\
22: 5 \\
22: 6\end{array}$ & $\begin{array}{l}0,0005 \\
0,025 \\
0,0005\end{array}$ & $\begin{array}{l}0,0025 \\
0,025 \\
0,01 \\
0,025 \\
0,0005 \\
0,0005\end{array}$ & $\begin{array}{l}0,0005 \\
0,005 \\
0,025 \\
0,005 \\
0,005 \\
\\
\\
0,0005 \\
0,025 \\
0,0025\end{array}$ & $\begin{array}{l}0,025 \\
0,0005 \\
0,0005 \\
0,0005 \\
0,01 \\
0,0005 \\
0,0025\end{array}$ & $\begin{array}{l}0,025 \\
0,0005 \\
0,0005 \\
0,0005 \\
0,0025 \\
0,0005 \\
0,0005 \\
0,0005 \\
0,0005 \\
0,0005\end{array}$ & $\begin{array}{l}0,0125 \\
0,05 \\
0,05 \\
0,0005 \\
0,0005 \\
0,0025 \\
0,0005 \\
0,0005\end{array}$ & $\begin{array}{l}0,0025 \\
0,005 \\
0,0005 \\
0,01 \\
0,0025 \\
0,0005 \\
0,0005 \\
0,005 \\
0,0005\end{array}$ & $\begin{array}{l}0,0005 \\
0,0025 \\
0,005 \\
0,0025 \\
0,0005 \\
0,0005 \\
0,0005 \\
0,0005 \\
0,0025\end{array}$ & $\begin{array}{l}0,0025 \\
0,0005 \\
0,0005 \\
0,01 \\
0,0025 \\
0,01 \\
0,0025 \\
0,0005 \\
0,0005 \\
0,01 \\
0,0005\end{array}$ \\
\hline
\end{tabular}

Tab. 6 Körpergewichte $(\bar{x} \pm s)$ in $g$ in der 9. und 12. Lebenswoche mit zugehörigen P-Werten der Körpergewichtsdifferenzen zwischen den mit gerad-

\begin{tabular}{|c|c|c|c|c|c|c|c|c|c|c|}
\hline \multirow{2}{*}{\multicolumn{2}{|c|}{$\begin{array}{c}\text { Nr. der Gruppe } \\
\text { Art des mittelkettigen } \\
\text { Triglycerids } \\
\% \text { Linolsäure in der Diät } \\
\text { Anzahl der Ratten pro } \\
\text { Gruppe }\end{array}$}} & \multicolumn{2}{|r|}{1,0} & $\stackrel{11}{\text { ungeradzahlig }}$ & $\stackrel{111}{\text { geradzahlig }}$ & 0,2 & $\stackrel{\text { IV }}{\text { ungeradzahlig }}$ & $\underset{\text { geradzahlig }}{\text { V }}$ & 0,0 & $\underset{\text { ungeradzahlig }}{\text { VI }}$ \\
\hline & & 9 & & 9 & 10 & & 10 & 10 & & 10 \\
\hline 9. Lebenswoche & $\begin{array}{l}\bar{x} \pm s \\
P\end{array}$ & $204,0 \pm 12,2$ & 0,15 & $197,7 \pm 11,6$ & $211,9 \pm 8,9$ & 0,45 & $211,9 \pm 11,9$ & $197,1 \pm 11,0$ & 0,0005 & $5212,0 \pm 9,3$ \\
\hline 12. Lebenswoche & $\begin{array}{l}\bar{x} \pm s \\
\mathbf{P}<\end{array}$ & $295,7 \pm 16,6$ & 0,001 & $256,1 \pm 16,2$ & $283,5 \pm 10,6$ & 0,48 & $282,7 \pm 19,9$ & $267,2 \pm 18,0$ & 0,1 & $278,6 \pm 12,2$ \\
\hline
\end{tabular}

zum einen zwischen Gruppen mit unterschiedlichem mittelkettigen Triglycerid-Bestandteil bei gleichem $\mathrm{Li}$ nolsäureanteil in der Diät auf, zum anderen zwischen Gruppen mit gleichem mittelkettigen Triglycerid-Bestandteil und unterschiedlichen Linolsäureanteilen in der Diät. Die Körpergewichtsanstiege (Wachstum) der Gruppen-(Tab. 6) wiesen zwei signifikante Unterschiede auf: in der 12. Lebenswoche lagen die Gewichte der mit geradzahligem mittelkettigem Triglycerid und $1 \%$ Linolsäure ernährten Tiere signifikant $(P<0,001)$ höher als die mit ungeradzahligem mittelkettigem Triglycerid und $1 \%$ Linolsäure aufgezogenen Tiere. Bei den ohne Linolsäure aufgezogenen Tieren kam ein Unterschied in der 9. Lebenswoche in umgekehrter Richtung heraus $(\mathrm{P}<0,0005)$, der in der 12. Lebenswoche nicht mehr vorhanden war. Weitere Daten über Wachstumsverhalten und andere biologische Parameter sollen in anderem Zusammenhang publiziert werden (BERNÀŠEK).

\section{Diskussion}

Bei Diätversuchen an Ratten ist die Fütterungstechnik von besonderer Bedeutung. Im Zusammenhang mit der Untersuchung des Stoffwechsels der essentiellen Fettsäuren wurde von mehreren Untersuchern eine fettfreie Basisdiät ad libitum verabreicht und die definierte Dosis an essentiellen Fettsäuren täglich per Sonde oral gegeben z. T. länger als 100 Tage lang $(13,14,15)$. Ein solches Fütterungṣverfahren stellt sicher, daß die beabsichtigte Dosis täglich aufgenommen wird. Ein derart aufwendiges Verfahren ist auch gut zu begründen, wenn es sich bei dem mit der Sonde zugeführten Stoff um einen solchen handelt, dessen Wirkung einzig von der täglich zugeführten Absolutmenge abhängt. Das ist jedoch bei der Linolsäure mit Sicherheit nicht der Fall. Das Fettsäuremuster der Organe resultiert aus dem Muster der den kettenverlängernden und olefinierenden Enzymsystemen angebotenen Fettsäuren, die nach dem Mechanismus der kompetitiven Hemmung in unterschiedlichen Raten kettenverlängert und/oder olefiniert werden (16-19). Für die Wirkung der Linolsäure auf das Fettsäuremuster kommt es also in erster Linie auf die Quotienten der molaren Konzentrationen der Linolsäure und der diversen am Enzym konkurrierenden Fettsäuren wie Ölsäure $\left(C_{18: 1}\right)$, Linolensäure $\left(C_{18: 3}\right)$ und wahrscheinlich noch der Palmitoleinsäure $\left(C_{16: 1}\right)$ an. Die nicht essentiellen Fettsäuren, vor allem Ölsäure und Palmitoleinsäure, werden teilweise oder in unserem Experiment schließlich sogar vollständig aus mittelkettigen Fettsäuren, Kohlenhydrat und Protein synthetisiert. Um die genannten Quotienten durch ein Diätregime über längere Zeit $\mathrm{zu}$ stabilisieren, ist es daher nach unserer Meinung am besten, eine in ihrer quantitativen und qualitativen Zusammensetzung definierte Diät ad libitum zu geben $(11 ; 20-22)$. Regelmäßige Gewichtskontrollen würden durch Stillstand oder Abnahme des Gewichts Hinweise geben, wenn eine nicht optimale Futteraufnahme beim einzelnen Versuchstier auftreten sollte.

Wir beschränkten uns auf die gaschromatographische Analyse der Gesamtfettsäuren der Leber aus zwei Gründen. Zum ersten ist bekannt $(11,21,24,25)$, daß unter Diäteinfluß die Organfettsäuremuster sich in den verschiedenen Lipidklassen zwar sehr unterschiedlich stark ausgeprägt, überall jedoch den gleichen Tendenzen folgend ändern, so daß weiter aufgefächerte Analysen in 
der Regel nicht adäquat dem Mehraufwand zusätzliche Information erbringen. Zweitens konnten von anderen Autoren (13-15) wiederum im Zusammenhang mit dem Stoffwechsel der essentiellen Fettsäuren gute Resultate allein aus der gaschromatographischen Analyse der Lebergesamtfettsäuren erzielt werden.

Alle angeführten Überlegungen unterstreichen überdies die Notwendigkeit, eine gewisse Größe der Versuchstiergruppen und der Anzahl gleicher Analysen nicht zu unterschreiten, damit die Stichhaltigkeit eventuell zu beobachtender Unterschiede mit statistischen Methoden geprüft werden kann.

Bei der Resorption aus dem Darmlumen verhält sich offenbar ungeradzahliges mittelkettiges Triglycerid nicht anders als geradzahliges. Die bisher zu der Frage vorliegenden Arbeiten $(26 ; 27)$ lassen jedenfalls keine Unterschiede erkennen. Im Gegensatz zu langkettigen Fettsäuren, die in der Darmepithelzelle ganz überwiegend zu Triglyceriden resynthetisiert und mit der Lymphe abtransportiert werden, gelangen die mittelkettigen Fettsäuren zu über 95\% als freie Fettsäuren ins Pfortaderblut und dann unmittelbar in die Leber.

Seit fast 60 Jahren sind Unterschiede im Stoffwechselverhalten von gerad- und ungeradzahligen kurzkettigen Fettsäuren bekannt. Bereits 1913 zeigte RINGer (29) am diabetischen Hund, daß Propionsäuregaben die Glucoseausscheidung steigern. Im Gegensatz zu den ketogen wirkenden geradzahligen Fettsäuren verhalten sich die ungeradzahligen Fettsäuren äquimolekular mit Propionsäure als Glycogenbildner (30). Weitere Aufschlüsse brachten Markierungsversuche (31). Nach Verabfolgung eines Gemisches aus Carboxyl-13 $\mathrm{C}$ - und $\gamma^{-14} \mathrm{C}-n$-Valerianat an Ratten war die Markierung des Glucosemoleküls im Leberglycogen mit ${ }^{14} \mathrm{C}$ identisch mit der in einem Parallelversuch, in dem entsprechend markierte Propionsäure gegeben wurde. Die gleichzeitig aufgetretene ${ }^{13} \mathrm{C}$ Narkierung entsprach der, die die Autoren in einem weiteren Parallelversuch mit markiertem Acetat erhielten. In einer erst kürzlich erschienenen Untersuchung (32) zeigten Ratten nach 4-6 Wochen unter einer Diät, die $27 \%$ der Kalorien als Triundecanin (Fettsäure $\mathrm{C}_{11: 0}$ ) enthielt, während einer daran anschließenden 2 bis 6tägigen Fastenperiode höhere Glycogenbestände in Leber und Muskulatur als Kontrollen.

Diese Ergebnisse belegen einwandfrei, daß die ungeradzahligen Fettsäuren zunächst ebenfalls unter Bildung von Acetatresten abgebaut werden und daß das schließlich anfallende Propionyl-CoA glycogenetisch wirkt. Es wird über u. a. Succinat in den Tricarbonsäurezyklus eingeführt $(33 \mathrm{a} ; \mathrm{b})$. Man kann also davon ausgehen, $\mathrm{da} B$ bei Zufuhr ungeradzahliger mittelkettiger Fettsäuren im Vergleich zur gleichen Menge geradzahliger mittelkettiger Fettsäuren der Pool an Acetyl-CoA der zur Kettenverlängerung, d. h. Bildung langkettiger Fettsäuren verfügbar ist, um 20 bis $30 \%$ kleiner sein wird. Gleichzeitig aber werden höhere Glycogenbestände vothanden sein und für die Energiegewinnung anstelle von Fettsäuren verfügbar sein. Welche Auswirkung als
Folge der Gabe ungeradzahliger im Vergleich zur Gabe geradzahliger Fettsäuren am Fettsäuremuster der Leber auftritt, wurde unseres Wissens bisher noch nicht geprüft.

Nach unseren früheren Untersuchungen (25) war zu erwarten, daß bei mittelkettiger Triglycerid-Fütterung die Auswirkungen auf das Fettsäuremuster der Organe je nach dem Grad der' Linolsäureversorgung unterschiedlich sein würden. Daher prüften wir die durch die Ungeradzahligkeit des mittelkettigen Triglycerids hervorgerufenen Unterschiede im Fettsäuremuster der Leber bei drei verschiedenen Versorgungszuständen mit Linolsäure.

Aus den Ergebnissen der Versuche lassen sich die Effekte der variierten zwei Faktoren teilweise getrennt analysieren. Der eine Faktor ist durch den Unterschied der mittelkettigen Triglycerid-Präparate gegeben. Der zweite Faktor wird wirksam in den unterschiedlichen Gaben an Linolsäure. Unterschiede der Linolsäureversorgung bewirken zahlreichere signifikante Differenzen der Leberfettsäuren als die Unterschiede des mittelkettigen Triglycerids (Tab.5). Stellt man die Daten der Tabelle 4 graphisch dar, so sind zwei Reaktionstypen auf die Linolsäureversorgung durch die Diät bei den einzelnen Fettsäuren der Leber zu unterscheiden. Bei $C_{14: 0}, C_{15: 0}, C_{16: 0}, C_{16: 1}, C_{18: 1}, C_{18: 3}$ und $C_{20: 3 n-9}$ beobachtet man im Linolsäuremangel höhere Werte, niedrigere Werte dagegen bei $C_{18: 0}, C_{18: 2}, C_{20: 3 n-6}, C_{20: 4}$, $\mathrm{C}_{22: 4}$ und $\mathrm{C}_{22: 5}$. (Zusf. Lit. (19)). Die Unterschiede in der Art des zugeführten mittelkettigen Triglycerids bewirken demgegenüber signifikante quantitative Differenzen nur im Ausmaß der Reaktion auf Linolsäuremangel. Bei $C_{18: 2}$ und $C_{20: 3 n-9}$ sind diese Unterschiede sehr ausgeprägt. Einzig im Falle von $C_{15: 0}$ kehrt sich der Reaktionstyp um. Mit den erwähnten Stoffwechseldifferenzen $z$ wischen gerad- und ungeradzahligen mittelkettigen Fettsäuren lassen sich die Befunde nur zum Teil erklären. Wenn man annimmt, daß das ungeradzahlige mittelkettige Triglycerid weniger Acetyl-CoA als das geradzahlige zur Kettenverlängerung zur Verfügung stellt, dann dürften $C_{16: 0}$ und $C_{16: 1}$ sowie im Linolsäuremangel auch $\mathrm{C}_{18: 1}$ davon besonders stark betroffen sein, denn sie zeigen unter ungeradzahligem mittelkettigem Triglycerid überwiegend signifikant niedrigere Werte. Auch $\mathrm{C}_{18: 2}$ liegt unter ungeradzahligem mittelkettigen Triglycerid bei ausreichender Linolsäureversorgung signifikant niedriger im Gegensatz zur $\mathrm{C}_{20: 4}$, die signifikant höher liegt, obwohl sie aus $\mathrm{C}_{18: 2}$ entsteht. Unterschiede der letztern beiden essentiellen Fettsäuren sind im Linolsäuremangel aber nicht mehr signifikant. Die ungeradzahlige Fettsäure $C_{15: 0}$ ist unter ungeradzahligem mittelkettigen Triglycerid besonders begünstigt, da sie durch Kettenverlängerung aus $C_{9: 0}$ entstehen dürfte, die sonst kaum zur Verfügung steht. $\mathrm{Daß}$ sie im Linolsäuremangel stark ansteigt, spricht dafür, daß dann verstärkt Kettenverlängerung aus mittelkettigen Fettsäuren stattfindet.

Die Einflüsse des ungeradzahligen mittelkettigen Triglycerids auf das Wachstum sind in unserer Serie un- 
einheitlich je nach Alter und Linolsäureversorgung. Weder aus den Unterschieden der Fettsäuremuster noch aus dem Wachstumsverhalten ergeben sich also Hinweise darauf, daß ungeradzahlige mittelkettige Fettsäuren weniger gut zur Ernährung geeignet sind als geradzahlige. Insbesondere liegen im Linolsäuremangel die Anteile an Linol- und Arachidonsäure nicht niedriger, die Werte für die Ölsäure nicht höher. Der TrienTetraen-Quotient (34) als Maßstab der Linolsäureversorgung liegt gerade bei fehlender Linolsäureversor- gung unter ungeradzahligem mittelkettigen Triglycerid günstiger als unter geradzahligem mittelkettigem Tryglycerid (Tab. 4). Es ist also auch im Hinblick auf die essentiellen Fettsäuren keinesfalls ein nachteiliger Unterschied festzustellen.

Für anregende Gespräche und freundliche Untcrstützung haben wir den Herren Prof. Dr. J. Kürnau, Prof. Dr. K. D. Vorgt, Prof. Dr. G. Brliek und Dipl. Chem. H. E. Rost sowie Frau Prof. Dr. H. Debuch zu danken. Frl. S. Kulow und Frl. D. HoRNung danken wir für zuverlässige technische Assistenz.

\section{Literatur}

1. Hashim, S. A., J. Amer. Diet. Ass. 51, 221 (1967). - 2. SiCKINGER, K., Dtsch. med. Wschr. 93, 1600 (1968). - 3. CREutZFELDT, W. und $K$. SICKINGER, Aktuelle Gastroenterologie, 24. Tagung, 141 (1968). - 4. FolCH, J., M. LeEs und G. H. Stoane-Stanley, J. biol. Chemistry 226, 497 (1957). - 5. Grimmer, G., A. Glaser, H. Oertel, K. D. Votgt und M. ApostoLAKIS, Hoppe-Seyler's Zschr. physiol. Chem. 333, 232 (1963). 6. Zö.LINER, N. und D. EBERHAGEN (Hsg.), Untersuchung und Bestimmung der Lipoide im Blut, Springer Verlag Berlin-Heidelberg-New York (1965). - 7. KAISER, R., Chromatographie in der Gasphase, Bd. IV, S. 121 u. 122, Bibliograph. Institut Mannheim (1965). - 8. WoOdFord, F. P. und C. M. van GENT, J. Lipid Res. 1, 188 (1960). - 9. Mrwa, T. K., K. L. Mikolajczak, F. R. EArLe und I. A. Wolf, Analytic. Chem. 32, 1739 (1960). 10. Hofstetrer, H. H., N. Sen und R. T. Holman, J. Amer. Oil Chemists' Soc. 42, 537 (1965). - 11. WALKER, B. L., J. Nutrit. 92, 23 (1967). - 12. Documenta GEIGY, Wiss. Tabellen 6. Aufl. - 13. Mohrhauer, H. und R. T. Holman, J. Lipid Res. 4, 151 (1963). - 14. Schlenk, H. und D. M. SAND, Biochim. biophysica Acta (Amsterdam) 144, 305 (1967). - 15. SPRECHER, H., Lipids 3, 14 (1968). - 16. Dhopeshwarkar, G. A. und J. F. MEAD, J. Amer. Oil Chemist's Soc. 38, 297 (1961). - 17. Holman, R. T. und H. Mohrhauer, Acta Chem. scand. 17, 84 (1963). - 18. Holman, R. T., Fed. Proc. 23, 1062 (1964). 19. Mead, J. F., in R. T. Holman (ED.): Progress in the che- mistry of fats and other lipids, Vol. 9, part 2, Pergamon Press Oxford-New York (1968). - 20. Stearns, E. M. Jr., J. A. Rysavy und O. S. Privett, J. Nutrit. 485 (1967). - 21. Walker, B. L., J. Nutrit. 94, 469 (1968). - 22. vaN Golde, L. M. G., W. A. Pieterson und L. L. M. van Deenen, Biochim. biophysica Acta (Amsterdam), 152, 84 (1968). - 23. CENrury, B., L. A. Witring, C. C. Harvey und M. K. Horwitr, Amer. J. Clin. Nutrit. 13, 362 (1962). - 24. Carroll, K. K., J. Amer. Oil Chemist's Soc. 42, 516 (1965). - 25. Seng, P. und J. BERNAšEK, Medizin und Ernährung 9, 149 (1968). - 26. HASHIM, S. A., K. Krell, P. Mao und T. B. van Itallie, Nature (London) 207, 527 (1965). - 27. LEE, D. S., S. A. HASHIM und T. B. vaN ITALlie, Amer. J. Physiol. 214, 294 (1968). - 28. Hyun, S. A., V. Vahouny und C. A. Treadwell, Biochim. biophysica Acta (Amsterdam) 137, 296 (1967). - 29. Ringer, A. J., J. biol. Chemistry 14, 43 (1913). - 30. Deuex, H. J. Jr., J. S. Butrs, L. F. Hallman und C. H. Cutler, J. biol. Chemistry 112, 15 (1935-36). - 31. Siegel, J. und V. Lorber, J. biol. Chemistry 189, 571 (1951). - 32. van Itallie, T. B. und A. K. KhachaDURIAN, Science Washington 165, 811 (1969). - 33a. MAZUMDER, R., T. Sasakawa und S. Ochoa, J. biol. Chemistry 238, 50 (1963). 33b. Cannata, J. J. B., A. Focesi Jt., R. Mazumder, R. C. Warner und S. Ochoa, J. biol. Chemistry 240, 3249 (1965). 34. Holman, R. T., J. Nutrit. 70, 405 (1960).

Dr. P. N. Seng $5 \mathrm{Köln} 41$

Joseph-Stelzmann-Str. 52 